\title{
Atuação do enfermeiro na reabilitação da saúde da pessoa com deficiência
}

\author{
Nurses performance in the health rehabilitation of persons with disabilities
}

Desempeño de las enfermeras en la rehabilitación de la salud de personas con discapacidad

Naara Rayssa Nascimento da Silva ${ }^{1 *}$, Amanda Cavalcante de Macêdo, Géssyca Cavalcante de Melo, Irena Penha Duprat ${ }^{1}$, Edivânia dos Santos Sobrinha ${ }^{1}$, Ana Lybia de Oliveira Bezerra da Silva ${ }^{1}$, Tales Luiz dos Santos Gomes ${ }^{1}$, Maria Clarisse Soares Carnaúba ${ }^{1}$, Amanda Priscilla Pereira Rocha ${ }^{1}$.

\section{RESUMO}

Objetivo: Descrever a atuação do enfermeiro na reabilitação de pessoas com deficiência. Métodos: Revisão integrativa de literatura. As bases consultadas foram Literatura Latino-Americana e do Caribe em Ciências da Saúde (LILACS), Base de Dados de Enfermagem (BDENF) e Scientific Electronic Library Online (SciELO). Incluídos artigos científicos que abordam a atuação do enfermeiro na reabilitação da saúde da pessoa com deficiência, escritos em português, inglês e espanhol, disponíveis gratuitamente. Não foi estabelecido limite temporal a fim de contemplar toda a produção até o momento. Excluídos artigos de reflexão, editoriais e revisão. A amostra final foi 18 artigos. Resultados: Estudos brasileiros totalizaram 83,3\% da amostra. Após análise, emergiram seis eixos relacionados à atuação do enfermeiro na reabilitação da pessoa com deficiência: enfermeiro como educador $(66,6 \%)$; enfermeiro como ser político e com compromisso social $(55,5 \%)$; enfermeiro como implementador de cuidados $(50 \%)$; enfermeiro como pesquisador $(27,7 \%)$; enfermeiro como mediador entre paciente, família e equipe multiprofissional $(22,2 \%)$; e enfermeiro como gerente da equipe de Enfermagem $(11,1 \%)$. Considerações Finais: $O$ enfermeiro atua de forma multidimensional no cenário relacionado à pessoa com deficiência, envolvendo além do cuidado direto, o gerenciamento de equipes, mediação do processo de trabalho em saúde, gestão através da implementação de políticas públicas e pesquisa científica.

Palavras-chave: Enfermagem, Pessoas com deficiência, Enfermagem em reabilitação.

\begin{abstract}
Objective: Describe the role of nurses in the rehabilitation of people with disabilities. Methods: Integrative literature review. The databases consulted were Latin American and Caribbean Literature on Health Sciences (LILACS), Nursing Database (BDENF) and Scientific Electronic Library Online (SciELO). Including scientific articles that address the role of nurses in the rehabilitation of the health of people with disabilities, written in Portuguese, English and Spanish, available for free. No time limit was established to contemplate all production to date. Reflection, editorial and review articles were excluded. The final sample was 18 articles. Results: Brazilian studies accounted for $83.3 \%$ of the sample. After analysis, six axes related to the role of the nurse in the rehabilitation of the person with disabilities emerged: nurse as an educator $(66.6 \%)$; nurse as a political being and with social commitment (55.5\%); nurse as a care implementer (50\%); nurse as a researcher (27.7\%); nurse as a mediator between patient, family and multiprofessional team (22.2\%); and nurse as manager of the Nursing team (11.1\%). Final Considerations: The nurse acts in a multidimensional way in the scenario related to people with disabilities, involving, in addition to direct care, team management, mediation of the health work process, management through the implementation of public policies and scientific research.
\end{abstract}

Keywords: Nursing, Disabled persons, Rehabilitation nursing.

1Universidade Estadual de Ciências da Saúde de Alagoas (UNCISAL), Maceió - AL.

*E-mail: naara.silva@academico.uncisal.edu.br

SUBMETIDO EM: 11/2020

ACEITO EM: 12/2020

PUBLICADO EM: 2/2021 


\section{RESUMEN}

Objetivo: Describir el papel del enfermero en la rehabilitación de personas con discapacidad. Métodos: Revisión integradora de la literatura. Las bases de datos consultadas fueron Literatura Latinoamericana y del Caribe en Ciencias de la Salud (LILACS), Base de Datos de Enfermería (BDENF) y Biblioteca Electrónica Científica en Línea (SciELO). Incluyendo artículos científicos que abordan el papel de las enfermeras en la rehabilitación de la salud de las personas con discapacidad, escritos en portugués, inglés y español, disponibles de forma gratuita. No se estableció límite de tiempo para contemplar toda la producción hasta la fecha. Se excluyeron los artículos de reflexión, editoriales y de revisión. La muestra final fue de 18 artículos. Resultados: Los estudios brasileños representaron el $83,3 \%$ de la muestra. Tras el análisis, surgieron seis ejes relacionados con el papel del enfermero en la rehabilitación de la persona con discapacidad: la enfermera como educadora (66,6\%); la enfermera como ser político y con compromiso social $(55,5 \%)$; enfermera como implementadora de cuidados (50\%); enfermera como investigadora (27,7\%); enfermera como mediadora entre paciente, familia y equipo multiprofesional (22,2\%); y enfermera como jefa del equipo de Enfermería $(11,1 \%)$. Consideraciones Finales: La enfermera actúa de forma multidimensional en el escenario relacionado con las personas con discapacidad, involucrando, además de la atención directa, la gestión de equipos, la mediación del proceso de trabajo en salud, la gestión a través de la implementación de políticas públicas y la investigación científica.

Palabras clave: Enfermería, Personas con discapacidad, Enfermería en rehabilitación.

\section{INTRODUÇÃO}

De acordo com o relatório da Organização das Nações Unidas (2018), existem mais de 1 bilhão de indivíduos com deficiência no mundo. Embora esse número seja bastante significativo, foram percebidas ainda muitas lacunas e desvantagens no que diz respeito à inclusão e acessibilidade dessas pessoas.

No último censo demográfico realizado pelo Instituto Brasileiro de Geografia e Estatística (IBGE), em 2010, quase 46 milhões de brasileiros, cerca de $24 \%$ da população, declarou ter algum grau de dificuldade em pelo menos uma das habilidades de enxergar, ouvir, caminhar ou subir degraus, ou possuir deficiência intelectual (IBGE, 2010). Tais deficiências podem acontecer de forma congênita ou serem adquiridas ao longo da vida com o processo de envelhecimento, por exemplo -, demandando intervenções através de reabilitação.

Segundo Leopardi MT, et al. (2014), o processo de reabilitação do indivíduo é complexo e está imerso em diversos aspectos biopsicossociais. Considerando-se esta complexidade, a pessoa com deficiência deve ser assistida por uma equipe multiprofissional capacitada e empenhada.

Além deste aspecto, o próprio indivíduo e sua família devem ser envolvidos nos esforços para a promoção de qualidade de vida, através do empoderamento e construção de alternativas a partir da percepção de suas potencialidades (LEOPARDI MT, et al., 2014)

Neste contexto, destaca-se a atuação dos trabalhadores em Enfermagem durante todo o processo de atenção à pessoa com deficiência e sua família, da atenção primária até os serviços de alta complexidade, na promoção, proteção e recuperação da saúde (ANDRADE LT, et al., 2010).

Embora existam relatos na literatura sobre a atuação de enfermeiros nesse segmento desde Florence Nightingale, ainda existe pouca produção científica no Brasile no mundo que impulsione está reflexão e colabore para a definição do papel do enfermeiro junto a este público (ANDRADE LT, et al., 2010).

Tendo em vista o número de pessoas com deficiência no Brasil e no mundo que demandam um cuidado integral, a escassez de estudos nesta temática, bem como a dificuldade ainda verificada de o enfermeiro reconhecer seu papel junto a este público, este estudo teve como objetivo descrevera atuação do enfermeiro na reabilitação da saúde pessoa com deficiência. 


\section{MÉTODOS}

Trata-se de uma revisão integrativa (RI) de literatura desenvolvida em 6 etapas (CARVALHO R, et al., 2010). Partiu-se da indagação: "o que diz a literatura científica sobre a atuação do enfermeiro na reabilitação da saúde da pessoa com deficiência? ". Foram consultadas as bases de dados: LILACS, BDENF e SciELO.

Foram incluídos artigos científicos que abordaram a atuação do enfermeiro na área de reabilitação, escritos em português, inglês ou espanhol, nacionais ou internacionais, disponíveis de forma gratuitas em limite temporal de publicação. Foram excluídos artigos que continham a metodologia descrita de forma incompleta e artigos de reflexão, editoriais e de revisão. A estratégia de busca foi adaptada para cada base de dados (Quadro 1).

O levantamento dos artigos científicos nas bases mencionadas foi realizado em junho de 2019. Foram obtidos na primeira busca um total de 154 artigos. Após verificação se correspondiam ao objeto a ser estudado, eliminação dos repetidos e se atendiam aos critérios de inclusão restaram 18 artigos, os quais compuseram a amostra.

Quadro 1 - Estratégias de busca utilizadas nas bases de dados e resultados obtidos.

\begin{tabular}{|c|c|c|c|c|}
\hline Base & Cruzamentos & $\mathbf{N}$ & $\begin{array}{c}\text { Artigos relacionados } \\
\text { ao Objetivo de } \\
\text { Pesquisa }\end{array}$ & $\begin{array}{c}\text { Artigos que } \\
\text { atenderam aos } \\
\text { critérios de inclusão }\end{array}$ \\
\hline SciELO & $\begin{array}{c}\text { Enfermagem [Todos os } \\
\text { índices] AND Deficiente OR Deficiência } \\
\text { [Todos os índices] AND Reabilitação } \\
\text { [Todos os índices] }\end{array}$ & 21 & 9 & 5 \\
\hline BDENF & $\begin{array}{c}\text { Enfermagem [Palavras] AND } \\
\text { Deficiente OR Deficiência [Palavras] } \\
\text { AND Reabilitação [Palavras] }\end{array}$ & 55 & 18 & 9 \\
\hline LILACS & $\begin{array}{c}\text { Enfermagem AND Deficiente OR } \\
\text { Deficiência AND Reabilitação }\end{array}$ & 78 & 20 & 4 \\
\hline & Total & 154 & 47 & 18 \\
\hline
\end{tabular}

Fonte: Silva NRN, et al., 2020.

Procedeu-se à caracterização dos artigos segundo o tipo, ano de publicação e nível de evidência. Vale salientar que o nível de evidência se trata de um critério de qualidade para trabalhos científicos, onde se realiza a análise crítica dos artigos. As revistas que adotam um padrão internacional utilizam 5 níveis de evidência, sendo o menor para relatos de caso e o maior para metanálise de trabalhos com randomização e seguimentos perfeitos (CAMANHO GL, 2009). O nível de evidência do estudo foi estabelecido conforme a Classificação de Oxford Centre for Evidence-Based Medicine (BADENOCH D, et al., 2009).

Para melhor visualização e compreensão, conforme recomendam Carvalho R, et al. (2010), os artigos selecionados foram divididos em subgrupos, baseados na temática geral do estudo "atuação do enfermeiro". Foi realizada a comparação entre todos os estudos selecionados e a identificação de padrões, diferenças e a sublocação desses tópicos para o delineamento dos eixos temáticos.

Através da conversão dos achados em subgrupos foram identificados seis eixos temáticos: 1 . Enfermeiro como educador; 2. Enfermeiro como ser político e com compromisso social;3. Enfermeiro como implementador de cuidados; 4. Enfermeiro como pesquisador; 5. Enfermeiro como mediador entre paciente, família e equipe multiprofissional; e 6 . Enfermeiro como gerente da equipe de enfermagem. Para compreensão dos resultados, os dados foram dispostos em quadros.

\section{RESULTADOS E DISCUSSÃO}

Os 18 artigos científicos selecionados foram caracterizados e ordenados pelo ano de publicação (Quadro 2) 
Quadro 2 - Síntese dos artigos científicos selecionados segundo o título, autores, periódico, ano, tipo de estudo e nível de evidência.

\begin{tabular}{|c|c|c|c|c|c|c|}
\hline $\mathbf{N}$ & Título & Autores & Periódico & Ano & Tipo de Estudo & NE* \\
\hline 1 & $\begin{array}{l}\text { Assistência ao binômio paciente/família na situação de } \\
\text { lesão traumática da medula espinhal }\end{array}$ & MANCUSSI AC & $\begin{array}{l}\text { Revista Latino-Americana } \\
\text { de Enfermagem }\end{array}$ & 1998 & Quantitativo e descritivo & $2 \mathrm{C}$ \\
\hline 2 & $\begin{array}{l}\text { O cuidar do enfermeiro especialista em reabilitação } \\
\text { físico-motora }\end{array}$ & $\begin{array}{l}\text { FARO ACM e LEITE } \\
\text { VBE }\end{array}$ & $\begin{array}{l}\text { Revista da Escola de } \\
\text { Enfermagem da USP }\end{array}$ & 2005 & Relato de Experiência & $4 C$ \\
\hline 3 & $\begin{array}{l}\text { Cuidado da criança com deficiência: suporte social } \\
\text { acessado pelas mães }\end{array}$ & $\begin{array}{l}\text { BARBOSA MAM,et } \\
\text { al }\end{array}$ & $\begin{array}{l}\text { Revista Gaúcha de } \\
\text { Enfermagem }\end{array}$ & 2009 & Qualitativo & $2 \mathrm{C}$ \\
\hline 4 & $\begin{array}{l}\text { Cuidar de crianças com necessidades especiais de } \\
\text { saúde: desafios para as famílias e enfermagem } \\
\text { pediátrica }\end{array}$ & $\begin{array}{l}\text { CABRAL IE e } \\
\text { NEVES ET }\end{array}$ & $\begin{array}{l}\text { Revista Eletrônica de } \\
\text { Enfermagem }\end{array}$ & 2009 & Qualitativo & $2 \mathrm{C}$ \\
\hline 5 & $\begin{array}{l}\text { Diagnósticos de enfermagem para o planejamento da } \\
\text { alta de homens prostatectomizados: um estudo } \\
\text { preliminar }\end{array}$ & CALDATO VG, et al & $\begin{array}{l}\text { Revista Eletrônica de } \\
\text { Enfermagem }\end{array}$ & 2009 & Quantitativo & $2 \mathrm{C}$ \\
\hline 6 & $\begin{array}{l}\text { Inclusão social da pessoa com deficiência: conquistas, } \\
\text { desafios e implicações para a enfermagem }\end{array}$ & $\begin{array}{l}\text { FRANÇA ISX e } \\
\text { PAGLIUCA LMF }\end{array}$ & $\begin{array}{l}\text { Revista da Escola de } \\
\text { Enfermagem da USP }\end{array}$ & 2009 & Qualitativo. & $2 \mathrm{C}$ \\
\hline 7 & $\begin{array}{l}\text { Processo de Enfermagem para pacientes com anorexia } \\
\text { nervosa. }\end{array}$ & RAMOS NA,et al & $\begin{array}{l}\text { Revista Brasileira de } \\
\text { Enfermagem }\end{array}$ & 2010 & Relato de Experiência & $4 \mathrm{C}$ \\
\hline 8 & $\begin{array}{l}\text { Identificando necessidades de crianças com deficiência } \\
\text { auditiva: uma contribuição para profissionais da saúde } \\
\text { e educação }\end{array}$ & DUPAS G,et al & $\begin{array}{l}\text { Escola Anna Nery Revista } \\
\text { de Enfermagem; }\end{array}$ & 2011 & Qualitativo & $2 \mathrm{C}$ \\
\hline 9 & $\begin{array}{l}\text { Vivencia de discapacidad por traumatismo de lamédula } \\
\text { espinal y elproceso de rehabilitación }\end{array}$ & $\begin{array}{c}\text { ECHEVERRÍA LG,et } \\
\text { al }\end{array}$ & Ciencia y Enfermería; & 2011 & Qualitativo & $2 \mathrm{C}$ \\
\hline
\end{tabular}




\begin{tabular}{|c|c|c|c|c|c|c|}
\hline 10 & $\begin{array}{l}\text { A pessoa estomizada e o processo de inclusão no } \\
\text { trabalho: contribuição para a enfermagem }\end{array}$ & MAURICIO VC & Rev. Bras. Enferm. & 2011 & Qualitativo & $2 \mathrm{C}$ \\
\hline 11 & $\begin{array}{l}\text { Mudanças na vida das pessoas com lesão medular } \\
\text { adquirida }\end{array}$ & $\begin{array}{l}\text { BITENCOURTRN, et } \\
\text { al }\end{array}$ & $\begin{array}{l}\text { Revista Eletrônica de } \\
\text { Enfermagem; }\end{array}$ & 2012 & Qualitativo & $2 \mathrm{C}$ \\
\hline 12 & $\begin{array}{l}\text { Corpo e corporalidade na paraplegia: significado das } \\
\text { mudanças }\end{array}$ & $\begin{array}{l}\text { FERGUSSON MEM } \\
\text { e REYMCPA }\end{array}$ & Avances em Enfermería. & 2012 & Qualitativo & $2 \mathrm{C}$ \\
\hline 13 & Qualidade de vida em pacientes com lesão medular & ALMEIDA PC,et al & $\begin{array}{l}\text { Revista Gaúcha de } \\
\text { Enfermagem; }\end{array}$ & 2013 & Quantitativo epidemiolog. & $2 \mathrm{C}$ \\
\hline 14 & $\begin{array}{l}\text { Perfil epidemiológico das crianças com paralisia } \\
\text { cerebral em atendimento ambulatorial }\end{array}$ & $\begin{array}{l}\text { CARVALHO ZMF,et } \\
\text { al }\end{array}$ & $\begin{array}{l}\text { Revista Enfermagem } \\
\text { UERJ }\end{array}$ & 2017 & Quantitativo & $2 \mathrm{C}$ \\
\hline 15 & $\begin{array}{l}\text { Percurso da pessoa com acidente vascular encefálico: } \\
\text { do evento à reabilitação }\end{array}$ & FARIA ACA,et al & $\begin{array}{l}\text { Revista Brasileira de } \\
\text { Enfermagem }\end{array}$ & 2017 & Qualitativo & $2 \mathrm{C}$ \\
\hline 16 & $\begin{array}{l}\text { Catálogo CIPE® para pacientes adultos em processo } \\
\text { de neurorreabilitação }\end{array}$ & ANDRADE LT & $\begin{array}{c}\text { Universidade Federal de } \\
\text { Minas Gerais; Belo } \\
\text { Horizonte; s.n; }\end{array}$ & 2018 & Quantitativo & $2 \mathrm{C}$ \\
\hline 17 & $\begin{array}{l}\text { A construção e validação de um aplicativo de } \\
\text { Enfermagem de reabilitação voltado a pessoas com } \\
\text { lesão medular e seus cuidadores sobre prevenção e } \\
\text { tratamento de lesões por pressão }\end{array}$ & ALVAREZ AB & $\begin{array}{l}\text { Escola de Enfermagem } \\
\text { Ana Nery. UFRJ }\end{array}$ & 2018 & Qualitativo & $2 \mathrm{C}$ \\
\hline 18 & Viver com deficiência física e o papel da rede de apoio & BOELL JEW, et al & $\begin{array}{l}\text { Revista de Enfermagem } \\
\text { do Centro Oeste Mineiro.- } \\
2018\end{array}$ & 2018 & Qualitativo & $2 \mathrm{C}$ \\
\hline
\end{tabular}

Legenda: *Nível de Evidência, Segundo a Classificação de Oxford Centre for Evidence-Based Medicine (BADENOCH D, et al., 2009).

Fonte: Silva NRN, et al., 2020. 
Das 18 publicações, $3(16,7 \%)$ foram internacionais, advindas do Chile, Colômbia e Portugal. Das publicações nacionais, $8(53,4 \%)$ foram desenvolvidas na região Sudeste do país. As regiões Nordeste e Sul contribuíram cada uma com $6(20 \%)$ das pesquisas, o Centro-oeste do Brasil, com 1 (6,6\%) e o Norte com nenhum estudo.

Destaca-se que os estudos de abordagem qualitativa representaram 55,5\% da amostra, com 10 estudos;17 (94,4\%) foram publicados em revistas de Enfermagem;4 (22,2\%) das publicações foram do ano de 2009.

Sobre o nível de evidência, 16 (88,9\%)dos estudos foram classificados como 2C - estudos de observação de resultados terapêuticos (outcomes research) (BADENOCH D, et al., 2009). Vale ressaltar que antes de 1998 não foram identificados estudos sobre este tema, bem como nos anos de 2019 e 2020 não houve produções sobre esta temática publicadas nas bases elencadas.

Para colaborar na análise, síntese e construção dos eixos temáticos, foi elaborado um quadro sinóptico (Quadro 3). 
Quadro 3 - Características dos artigos quanto aos objetivos, resultados e conclusão.

\begin{tabular}{|c|c|c|c|}
\hline $\mathbf{N}$ & Objetivos & Resultados & Conclusão \\
\hline 1 & $\begin{array}{l}\text { Identificar e analisar as intervenções de } \\
\text { enfermagem direcionadas à família ou ao } \\
\text { binômio paciente/família }\end{array}$ & $\begin{array}{l}\text { Foram identificados } 14 \text { diagnósticos de enfermagem } \\
\text { e } 183 \text { intervenções pertinentes a eles. }\end{array}$ & $\begin{array}{l}\text { Acredita-se ser necessário manter um canal de } \\
\text { comunicação com a família desde o momento do } \\
\text { trauma inserindo esta unidade de suporte no } \\
\text { processo de reabilitação. }\end{array}$ \\
\hline 2 & $\begin{array}{l}\text { Descrever as atividades desenvolvidas pelo } \\
\text { enfermeiro de reabilitação em uma unidade de } \\
\text { internação de pacientes em reabilitação físico- } \\
\text { motora e destacar sua importância frente à } \\
\text { equipe de reabilitação. }\end{array}$ & $\begin{array}{l}\text { Dentre as principais atividades desenvolvidas tem-se } \\
\text { a manutenção das necessidades fisiológicas, onde a } \\
\text { equipe de Enfermagem executa, orienta, demonstra, } \\
\text { supervisiona e reavalia as condições de autocuidado } \\
\text { destes pacientes. O enfermeiro atua, também, na } \\
\text { prevenção de possíveis complicações que podem } \\
\text { ocorrer frente à suscetibilidade peculiar desta clientela }\end{array}$ & $\begin{array}{l}\text { O enfermeiro reabilitador desenvolve um papel } \\
\text { fundamental de Educador, bem como de } \\
\text { Implementador de cuidados, Conselheiro e } \\
\text { Consultor, muitas vezes o responsável pelo } \\
\text { planejamento geral de reabilitação. }\end{array}$ \\
\hline 3 & $\begin{array}{l}\text { Compreender como a mãe de crianças com } \\
\text { deficiência atendidas em uma instituição de } \\
\text { reabilitação identifica e acessa as fontes de } \\
\text { suporte social para atender as necessidades } \\
\text { de seu filho e de sua família. }\end{array}$ & $\begin{array}{l}\text { Emergiram seis categorias temáticas representativas } \\
\text { da experiência da mãe ao acessar as redes de } \\
\text { suporte social, dentre elas o apoio da família, dos } \\
\text { amigos, esclarecimentos sobre a condição do filho, } \\
\text { suporte material e apoio da instituição especializada. }\end{array}$ & $\begin{array}{l}\text { O enfermeiro deve ter compromisso com essas } \\
\text { famílias, ajudando-as a compreender cada fase } \\
\text { vivenciada por elas }\end{array}$ \\
\hline 4 & $\begin{array}{l}\text { Descrever e discutir os desafios determinados } \\
\text { por esses cuidados para as cuidadoras de } \\
\text { CRIANES no domicílio. }\end{array}$ & $\begin{array}{l}\text { As mulheres desvelaram a natureza complexa do } \\
\text { cuidado às CRIANES e a relevância de realizá-lo com } \\
\text { base em saberes e práticas que não pertencem ao } \\
\text { seu cotidiano existencial. Elas situaram esse cuidado } \\
\text { como de sobrevivência, (sobre)natural e singular } \\
\text { devido a sua complexidade, elevada demanda e } \\
\text { dedicação. }\end{array}$ & $\begin{array}{l}\text { Conclui-se que é preciso incluir as famílias das } \\
\text { CRIANES na assistência de enfermagem no } \\
\text { intuito de ampliar o poder de negociação dessas } \\
\text { mulheres para além da sobrevivência. }\end{array}$ \\
\hline 5 & $\begin{array}{l}\text { Identificar diagnósticos de enfermagem em } \\
\text { pacientes prostatectomizados com vistas a } \\
\text { oferecer subsídios para a elaboração de planos } \\
\text { de cuidados para a alta hospitalar. }\end{array}$ & $\begin{array}{l}\text { Os diagnósticos identificados em todos os pacientes } \\
\text { foram: conhecimento deficiente } \\
\text { (acerca dos cuidados pós-operatórios e com sonda } \\
\text { vesical de demora) risco de volume de líquidos } \\
\text { deficiente, risco de lesão, risco de infecção, } \\
\text { integridadetissular prejudicada. }\end{array}$ & $\begin{array}{l}\text { Os diagnósticos identificados possibilitaram } \\
\text { realizar um detalhamento das condições dos } \\
\text { sujeitos e contribuíram com a realização de } \\
\text { discussão sobre as necessidades dos mesmos }\end{array}$ \\
\hline 6 & $\begin{array}{l}\text { Compreender o discurso das pessoas com } \\
\text { deficiência acerca da concretude do processo } \\
\text { de inclusão social e articular o discurso desses } \\
\text { sujeitos com as diretrizes do decreto no } \\
\text { 3.298/99. }\end{array}$ & $\begin{array}{l}\text { Esses sujeitos conquistaram legislação que assegura } \\
\text { direitos de cidadania, mas existem desafios no campo } \\
\text { de saúde, educação, profissionalização e da inserção } \\
\text { no mercado de trabalho }\end{array}$ & $\begin{array}{l}\text { Os enfermeiros reabilitadores precisam conhecer } \\
\text { a vivência desses sujeitos e dos seus familiares } \\
\text { para ajudá-los a buscar estratégias de } \\
\text { enfrentamento dos problemas que afetam sua } \\
\text { inclusão, e, consequentemente a sua saúde. }\end{array}$ \\
\hline
\end{tabular}




\begin{tabular}{|c|c|c|c|}
\hline $\mathbf{N}$ & Objetivos & Resultados & Conclusão \\
\hline 7 & $\begin{array}{l}\text { O objetivo deste estudo é relatar a experiência } \\
\text { da aplicação do PE a uma paciente com } \\
\text { anorexia nervosa utilizando os diagnósticos de } \\
\text { enfermagem da Taxonomia II da NANDA, as } \\
\text { intervenções de enfermagem da NIC e os } \\
\text { resultados de enfermagem da NOC. }\end{array}$ & $\begin{array}{l}\text { A partir da aplicação do Processo de Enfermagem, os } \\
\text { resultados planejados foram alcançados, } \\
\text { proporcionando melhor qualidade de vida, no período } \\
\text { em que permaneceu internada. }\end{array}$ & $\begin{array}{l}\text { Os fatores psíquicos, neurológicos, endócrinos e } \\
\text { imunológicos, peculiares na anorexia nervosa, } \\
\text { propiciaram a elaboração de um Processo de } \\
\text { Enfermagem, que contribuiu de maneira positiva } \\
\text { para a complementação da reabilitação da saúde } \\
\text { da mesma. }\end{array}$ \\
\hline 8 & $\begin{array}{l}\text { Identificar crianças em idade escolar, } \\
\text { matriculadas no ensino fundamental da rede } \\
\text { pública do município, conhecer o tipo de } \\
\text { acompanhamento de saúde que recebem e } \\
\text { identificar junto à família as necessidades que } \\
\text { vivenciam em decorrência da deficiência } \\
\text { auditiva. }\end{array}$ & $\begin{array}{l}\text { A família sente falta de suporte e infraestrutura do } \\
\text { município para diagnóstico e reabilitação, além } \\
\text { da falta de recursos e investimento no contexto } \\
\text { escolar, sendo obrigada a se mobilizar para enfrentar } \\
\text { essa situação. }\end{array}$ & $\begin{array}{l}\text { Destacamos a importância do enfermeiro diante } \\
\text { das ações de prevençãoprimária da deficiência } \\
\text { auditiva. }\end{array}$ \\
\hline 9 & $\begin{array}{l}\text { Descrever as experiências de pessoas com } \\
\text { deficiência devido a uma lesão na medula } \\
\text { espinhal. }\end{array}$ & $\begin{array}{l}\text { A pesquisa descreve a experiência de ter uma } \\
\text { deficiência devido a danos na medula espinhal, como } \\
\text { é difícil assumir essa condição e se preparar para a } \\
\text { reabilitação. Os autores descrevem as etapas do } \\
\text { processo de reabilitação; desde uma fase marcada } \\
\text { pela depressão, até atingir a independência absoluta } \\
\text { nos melhores casos. }\end{array}$ & $\begin{array}{l}\text { A presença do profissional de enfermagem é } \\
\text { uma constante nos diversos momentos do } \\
\text { caminho de aceitação da deficiência e } \\
\text { reabilitação que os informantes descrevem, } \\
\text { portanto, o papel do enfermeiro e os efeitos de } \\
\text { seu cuidado são importantes. }\end{array}$ \\
\hline 10 & $\begin{array}{l}\text { Identificar as facilidades e dificuldades dos } \\
\text { clientes ostomizados para inclusão no mundo } \\
\text { do trabalho; analisar as possibilidades de } \\
\text { inclusão no mundo do trabalho; discutir, a partir } \\
\text { do ponto de vista do cliente ostomizado, as } \\
\text { orientações fornecidas pelos enfermeiros com } \\
\text { vistas à inclusão no mundo do trabalho. }\end{array}$ & $\begin{array}{l}\text { Os resultados revelaram que a maioria dos sujeitos } \\
\text { trabalhava informalmente e recebia ao mesmo tempo } \\
\text { algum auxílio governamental. Ressalta-se que eles } \\
\text { reconheciam a ilegalidade dessa situação, porém, } \\
\text { julgavam-na necessária devido aos baixos valores } \\
\text { dos benefícios. }\end{array}$ & $\begin{array}{l}\text { Concluiu-se que o retorno ao trabalho foi } \\
\text { considerado essencial, mas existem inúmeras } \\
\text { dificuldades para este retorno e manutenção no } \\
\text { universo laboral. }\end{array}$ \\
\hline 11 & $\begin{array}{l}\text { Compreender quais são os impactos da lesão } \\
\text { medular na vida da pessoa por ela acometida. }\end{array}$ & $\begin{array}{l}\text { A pessoadivide sua vida entre antes, durante e depois } \\
\text { da lesão, revivendo o momento gerador diversas } \\
\text { vezes. A família é essencial na(re)adaptação da } \\
\text { pessoa. }\end{array}$ & $\begin{array}{l}\text { O enfermeiro deve conhecer mais sobre a } \\
\text { lesãomedular, suas intercorrências, e as formas } \\
\text { deintervenções possíveis, apostando sempre na } \\
\text { capacidade que o outro possui e na vida que há } \\
\text { pela frente. }\end{array}$ \\
\hline 12 & $\begin{array}{l}\text { Descrever os significados que eles têm para } \\
\text { pessoas com paraplegia, secundária a lesão } \\
\text { traumática da medula espinhal, alterações do } \\
\text { corpo e corporalidade. }\end{array}$ & $\begin{array}{l}\text { Análise de dados mostra que, com o tempo, as } \\
\text { pessoas se conscientizam. A partir das sequências, } \\
\text { eles aprendem a reconhecer novos padrões de } \\
\text { expressão de seu corpo, desenvolvendo novas } \\
\text { habilidades. }\end{array}$ & $\begin{array}{l}\text { Os resultados norteiam profissionais de saúde } \\
\text { em geral e enfermeiros em particular, por um } \\
\text { cuidado voltado para o re-conhecimento do novo } \\
\text { corpo e a reconstrução de uma nova } \\
\text { corporalidade. }\end{array}$ \\
\hline
\end{tabular}




\begin{tabular}{|c|c|c|c|}
\hline $\mathbf{N}$ & Objetivos & Resultados & Conclusão \\
\hline 13 & $\begin{array}{l}\text { Medir a qualidade de vida de adultos com lesão } \\
\text { medular e identificar os domínios que } \\
\text { prejudicam a qualidade de vida desses sujeitos }\end{array}$ & $\begin{array}{l}\text { Os resultados expressam a insatisfação dos } \\
\text { investigados com aqualidade de vida. }\end{array}$ & $\begin{array}{l}\text { O enfermeiro deve contribuir para a reabilitação } \\
\text { e reinclusão social da pessoa com lesão } \\
\text { medular, respeitando suas limitações, } \\
\text { enfatizando o potencial remanescente e a } \\
\text { capacidade para autocuidado. }\end{array}$ \\
\hline 14 & $\begin{array}{l}\text { Descrever o perfil de pais e crianças com } \\
\text { paralisia cerebral assistidas em ambulatórios } \\
\text { de Fortaleza, Ceará }\end{array}$ & $\begin{array}{l}\text { Identificou-se } 88(72,1 \%) \text { das crianças com PC } \\
\text { espástica, tendo como principal causa a anóxia } \\
\text { neonatal } 64(52,5 \%) \text {, em que foram diagnosticadas } \\
\text { com menos de um mês de vida } 47(38,5 \%) \text { e que além } \\
\text { do aspecto motor, apresentam outros distúrbios, } \\
\text { como problemas na fala } 92(75,4 \%) \text {. }\end{array}$ & $\begin{array}{l}\text { O enfermeiro deve identificar precocemente } \\
\text { essas alterações clínicas, por ocasião da } \\
\text { consulta de puericultura, fazer os devidos } \\
\text { encaminhamentos para equipe multiprofissional } \\
\text { e acompanhamento periódico a fim de que haja } \\
\text { uma intervenção o mais precoce possível }\end{array}$ \\
\hline 15 & $\begin{array}{l}\text { Descrever o percurso da pessoa com Acidente } \\
\text { Vascular Encefálico e identificar os } \\
\text { acontecimentos significativos neste percurso. }\end{array}$ & $\begin{array}{l}\text { Os dados revelaramque o trajeto da pessoa vai desde } \\
\text { o reconhecimento dos sintomas até a preparação da } \\
\text { alta hospitalar. A dependência traz a necessidade de } \\
\text { adquirir competências para se adaptar à nova } \\
\text { situação. }\end{array}$ & $\begin{array}{l}\text { Espera-se contribuir para a } \\
\text { melhoria dos cuidados de enfermagem no } \\
\text { atendimento das pessoas acometidas com } \\
\text { Acidente Vascular Encefálico, desde o } \\
\text { acometimento, recuperação e reabilitação, até } \\
\text { aos cuidados domiciliares. }\end{array}$ \\
\hline 16 & $\begin{array}{l}\text { Construir um Catálogo de conceitos da } \\
\text { Classificação Internacional para a Prática de } \\
\text { Enfermagem (CIPE®) na neurorreabilitação de } \\
\text { adultos. }\end{array}$ & $\begin{array}{l}\text { Foram construídos } 1490 \text { enunciados, sendo } 394 \\
\text { diagnósticos/resultados de enfermagem e } 1090 \\
\text { intervenções de enfermagem. Validou-se quanto ao } \\
\text { conteúdo } 266 \text { diagnósticos/resultados de } \\
\text { enfermagem e } 540 \text { intervenções. }\end{array}$ & $\begin{array}{l}\text { Desenvolveu-se um Catálogo } \\
\text { CIPE® para Adultos em processo de } \\
\text { neurorreabilitação à luz do modelo teórico de } \\
\text { adaptação de Roy. Diagnósticos / resultados e } \\
\text { intervenções de enfermagem foram propostos e } \\
\text { validados e para ajudar o raciocínio clínico do } \\
\text { enfermeiro. }\end{array}$ \\
\hline 17 & $\begin{array}{l}\text { Desenvolver uma tecnologia de informação } \\
\text { junto a pessoa com lesão medular e seus } \\
\text { cuidadores por meio de um aplicativo como } \\
\text { material educativo voltado a prevenção e } \\
\text { tratamento das lesões por pressão. }\end{array}$ & $\begin{array}{l}\text { Identificou-se questões que interferem diretamente no } \\
\text { cuidado como a necessidade da Rede de Cuidados à } \\
\text { pessoa com Deficiência, essencial em todo este } \\
\text { processo para a promoção da autonomia e qualidade } \\
\text { de vida da pessoa com lesão medular. }\end{array}$ & $\begin{array}{l}\text { O grau de concordância do aplicativo foi de } \\
91,7 \% \text {. O aplicativo foi avaliado como um recurso } \\
\text { positivo e } \\
\text { importante pelos participantes do estudo a ser } \\
\text { disseminado para outras pessoas. }\end{array}$ \\
\hline 18 & $\begin{array}{l}\text { Compreender o impacto da deficiência física na } \\
\text { pessoa e a contribuição da rede de apoio. }\end{array}$ & $\begin{array}{l}\text { Os dados foram organizados em três } \\
\text { categoriastemáticas: } \\
\text { autonomia/independência; mudanças no cotidiano e } \\
\text { no viver; e rede como apoio para superar/enfrentar as } \\
\text { dificuldades. }\end{array}$ & $\begin{array}{l}\text { A deficiência física apresenta transformações na } \\
\text { vida das pessoas, sendo a rede de apoio a elas, } \\
\text { peça fundamental para o enfrentamento das } \\
\text { dificuldades. }\end{array}$ \\
\hline
\end{tabular}

Fonte: Silva NRN, et al., 2020. 
Após a análise, emergiram seis eixos que englobam as perspectivas de atuação do enfermeiro na área de reabilitação da pessoa com deficiência, são eles: Enfermeiro como educador, mencionado em 12 (66,6\%) dos artigos; Enfermeiro como ser político e com compromisso social, em 10 (55,5\%); Enfermeiro como implementador do cuidado, em 09(50\%); Enfermeiro como pesquisador, em 5 (27,7\%); Enfermeiro como mediador entre paciente, família e equipe multiprofissional em $4(22,2 \%)$ e Enfermeiro como gerente da equipe de Enfermagem, em 2 (11,1\%). Para melhor compreensão, tais eixos de atuação foram detalhados nas subseções que se seguem, embasados pelas argumentações dos artigos da amostra e discutidos com o suporte da literatura.

\section{Enfermeiro como educador}

De acordo com os autores, seja em ambiente domiciliar, ambulatorial, comunitário ou hospitalar, o enfermeiro tem sido responsável pela reeducação do indivíduo com base em suas novas condições, buscando promover adaptação e desenvolvimento das potencialidades, promovendo independência funcional e prevenção de complicações secundárias (ALMEIDA PC, et al., 2013; FARO ACM e LEITE VBE, 2005; FRANÇA ISX e PAGLIUCA LMF, 2009).

As ações educativas têm sido desempenhadas por meio de orientações ao indivíduo e família sobre o autocuidado, com foco nas atividades de vida diária do indivíduo, sobretudo, quanto à higiene corporal, alimentação, hidratação, vestuário, aparência pessoal, eliminações, atividades do lar e o encorajamento dos sentimentos negativos, estando atento ainda para a prevenção de agravos e promoção da saúde (FARO ACM e LEITE VBE, 2005; FRANÇA ISX e PAGLIUCA LMF, 2009).

Os autores ponderam que uma atenção especial deve ser dada ainda às questões sociais, psicológicas e espirituais do binômio paciente-família o que exige uma visão holística, tratando o indivíduo como ser indissociável, não privilegiando uma dimensão em detrimento de outra (FARO ACM e LEITE VBE, 2005; FRANÇA ISX e PAGLIUCA LMF, 2009; MAURICIO VC, 2011). Os processos educativos, como grande compromisso do enfermeiro, contemplam não só o paciente e família, mas também a equipe e cuidadores. Isso gera mudança e formação de novos conceitos e por fim, transformação da realidade (BITENCOURT RN, et al., 2012).

Para compreender a ação do enfermeiro como educador na promoção da saúde e prevenção de agravos, pode-se tomar como exemplo a abordagem frente à prevenção da deficiência auditiva, na qual o profissional permanece em contato através da vacinação da gestante contra a rubéola e acompanhamento da criança para a eliminação de outros fatores de risco, além das orientações à família e à população em geral (DUPAS G, et al., 2011).

Conforme descreve o estudo de Araújo CAC e Caldas AJ (2020), o enfermeiro é percebido como responsável pela orientação dos indivíduos no âmbito físico, social e comportamental. As pesquisas são convergentes quanto ao papel fundamental do enfermeiro para o desenvolvimento de atividades educativas. Couto GR, et al. (2019) trouxeram em seu estudo que cerca de um quinto dos registros de enfermagem analisados em sua pesquisa traziam ações como "informar" e "gerir" nas intervenções, enquanto em cerca de $49,2 \%$ as ações eram do tipo "observar". Os números apontam para o déficit nas ações educativas ou na documentação das intervenções.

\section{Enfermeiro como ser político e com compromisso social}

A adaptação da pessoa e da família à nova realidade tem sido relatada como complexa e, para satisfazer às necessidades do binômio paciente-família, considerando o indivíduo como um todo indissociável, o enfermeiro é responsável pela mobilização de recursos da comunidade a fim de reintegrar a pessoa no ambiente social (FARIA ACA, et al., 2017). Tais mobilizações envolvem desde intervenções e adaptação da casa do paciente, por exemplo, até a participação na construção e na efetivação das políticas públicas para pessoas deficientes (CARVALHO ZMF, et al., 2017; FARIA ACA, et al., 2017).

França ISX e Pagliuca LMF (2009) apontam que o enfermeiro tem buscado conhecer e compreender a realidade e vivência dos indivíduos e de sua família, com o objetivo de assim auxiliá-los no enfrentamento dos desafios existentes para sua inclusão social. Para que a reabilitação seja baseada e fortalecida na comunidade, 0 enfermeiro deve ser protagonista reconhecido pela comunidade que está inserido (ECHEVERRÍA LG, et al., 2011). 
Nesse cenário, considera-se necessário que o profissional esteja guarnecido de conhecimento acerca das políticas públicas e decretos como o de no 3.298/99, diretriz que versa sobre a educação inclusiva que respalda o indivíduo deficiente, de modo a assegurar seus direitos, preservar suas funções existentes, compensar capacidades perdidas, descobrir novas potencialidades e facilitar os reajustes sociais (FRANÇA ISX e PAGLIUCA LMF, 2009).

O tipo de assistência que a pessoa deficiente tem demandado relaciona-se ao estabelecimento de uma rede de apoio, que envolve a família, a comunidade e a equipe de reabilitação para que se promova a (re)inclusão social e melhoria da qualidade de vida dos indivíduos (ALMEIDA PC, et al., 2013). Como membro da equipe multiprofissional, ao enfermeiro cabe promover beneficiar a implantação de serviços que atuem rumo ao alcance da independência e da melhoria das condições de vida do indivíduo, bem como da sua família, com ênfase na integração social (FARO ACM, 2006).

A enfermagem é compreendida como a profissão que possui como princípios, o saber técnico, científico, ético e cultural (SALES JNF, 2019). Andrade LT, et al. (2010) pontuam que só as competências técnicas não serão suficientes para contemplar todas as necessidades do indivíduo, portanto, os serviços devem buscar a reintegração da pessoa com base no planejamento, coordenação e avaliação das suas necessidades físicas, sociais, políticas e econômicas a fim de ressignificar as experiências vivenciadas pelos indivíduos (SALES JNF, 2019).

\section{Enfermeiro como implementador do cuidado}

O enfermeiro foi descrito por Faro ACM e Leite VBE (2005) e Andrade LT, et al. (2018) como o responsável pela elaboração do plano de cuidados de enfermagem e por coordenar planos de cuidados interdisciplinares. Ele deve buscar o distanciamento do empirismo, e embasar-se em metodologias de trabalho e ações cientificamente comprovadas (RAMOS NA, et al., 2010). A Sistematização da Assistência de Enfermagem (SAE) tem sido apontada como estratégia potencial para contribuir para a organização do Processo de Enfermagem (PE), a qual deve-se aplicar cada etapa com base em um referencial teórico (FERGUSSON MEM e REY MCPA, 2012; MAURICIO VC, 2011).

No estudo de Ramos NA, et al. (2010), realizado de junho a agosto de 2008, junto a um indivíduo com anorexia nervosa internado na unidade de psiquiatria foi aplicado o PE a partir do levantamento dos dados objetivos e subjetivos, sendo elaborado um plano de cuidados com base nos diagnósticos de enfermagem identificados, associados a questões biopsicossociais do indivíduo. As ações foram planejadas e os resultados foram exitosos, já que os objetivos e metas foram alcançados, o que proporcionou ao indivíduo melhor qualidade de vida. Tal fato destaca a crucial idade da adoção e apropriação de metodologias de trabalho para a implementação de cuidados no fazer do enfermeiro na reabilitação (RAMOS NA, et al., 2010).

A elaboração do Plano de Cuidados de forma adequada garante um detalhamento nas condições de saúde dos sujeitos e a promoção de um cuidado especializado e individualizado (CALDATO VG, et al., 2009). Couto GR, et al. (2019) salientam que a forma como o enfermeiro registra suas práticas diz muito sobre os ganhos e avanços reais para a enfermagem, embora Andrade LT, et al. (2010) descrevam ainda diversas lacunas existentes na documentação de enfermagem, no que diz respeito à falta de especificidade e presença de ambiguidade nos registros.

No estudo de Couto GR, et al. (2019), realizado em um centro de reabilitação em Portugal, foram percebidas muitas limitações nos registros de enfermagem no que diz respeito à coerência entre os diagnósticos e as intervenções, revelando confusão na documentação e quanto às reais necessidades dos indivíduos. Além disso, pouquíssimas intervenções foram registradas, esbarrando-se no ponto que: ou as intervenções não são implementadas ou não são documentadas.

Considerando o campo de reabilitação para os trabalhadores em Enfermagem no Brasil, acredita-se que os números não sejam animadores, já que ainda há a baixa inserção, valorização e apropriação do enfermeiro nesse cenário; logo, ainda não se conhece até que ponto o enfermeiro implementa os cuidados e os registra adequadamente. Esse ponto está em aberto para futuros estudos e reflexões em busca de compreender como se dá esse aspecto. 


\section{Enfermeiro como pesquisador}

Embora pouco explanada e desenvolvida nos estudos, essa categoria emergiu dos artigos analisados, no que se diz respeito à necessidade de aprimoramento na área da enfermagem em reabilitação.

Andrade LT, et al. (2018) aponta como dever do enfermeiro reabilitador o compromisso de constante aperfeiçoamento de suas competências para que se haja melhoria nos resultados alcançados pelos pacientes. Para tanto, o enfermeiro deve desenvolver estudos que busquem compreender melhor o processo de enfermagem e a elaboração de diagnósticos e intervenções para pessoas em reabilitação, bem como suas famílias (MANCUSSI AC, 1998).

Boell JEW, et al. (2018) descreve que a contribuição do profissional enfermeiro se refere tanto à prática, por meio da implementação de cuidados de enfermagem, como também pela contribuição teórica, por meio de estudos que proporcionem diferentes evidências que embasem e auxiliem a prática e as pessoas com deficiência em seus enfrentamentos.

Autores como Almeida PC, et al. (2013) e Alvarez AB (2018) salientam que os enfermeiros devem desenvolver estudos que validem instrumentos de avaliação e sejam incrementados na assistência sistematizada de enfermagem, contribuindo com novas tecnologias e estratégias válidas para o cuidado.

Conforme apontado no estudo de Leite JL, et al. (2019), a enfermagem quanto "ciência em construção" é recente e o distanciamento entre o enfermeiro que assiste e o que pesquisa ainda é grande, porém a enfermagem moderna cada vez mais tem exigido a construção e o consumo de pesquisas científicas, bem como a sua inserção no processo de trabalho.

Especificamente no campo da reabilitação, Azevedo PMDS, et al. (2019) evidenciam, por exemplo, a necessidade do desenvolvimento de ferramentas de avaliação para o autocuidado de forma mais ampla e significativa para a reabilitação.

Araújo CAC e Caldas AJA (2020) trazem em seu estudo que, para o alcance de uma prática baseada em evidência e fundamentada teoricamente, é responsabilidade do enfermeiro especialista em reabilitação a constante atualização científica e o fortalecimento do julgamento crítico, o que se traduz como dever de todo profissional de saúde aprofundar seus conhecimentos para melhorar seus resultados.

De forma geral, esse campo para a enfermagem tem trazido ainda muitas inquietações. Observa-se uma valorização dos profissionais médicos, fisioterapeutas, fonoaudiólogos e terapeutas ocupacionais, por exemplo, em detrimento do enfermeiro, já que aqueles, a partir de suas lutas conquistaram o devido espaço e reconhecimento, enquanto esses ainda seguem em luta.

\section{Enfermeiro como mediador entre o paciente, a família e a equipe multiprofissional}

Autores como Mancussi VC (1998) sugerem que os cuidados e intervenções de enfermagem, bem como da equipe de reabilitação, devem ser voltados ao paciente e à família, onde devem predominar ações de orientação e treinamento. Dessa forma, diante de tantas demandas, deve ser estabelecido e mantido canal de comunicação com a pessoa, família e equipe.

Cabral IE e Neves ET (2009) trazem que é necessário que os cuidados de enfermagem incluam as famílias para o compartilhamento de saberes e práticas junto à equipe. Segundo Bitencourt NR, et al. (2012), garantir relações integradoras entre o paciente e família nas redes de apoio, possibilita o crescimento pessoal e efetividade da reabilitação.

É dever do enfermeiro firmar compromisso com as famílias e desenvolver vínculo de confiança com elas, direcioná-las para a busca do apoio necessário dentro da rede de apoio e fazer os devidos encaminhamentos para a equipe multiprofissional, já que está em contato direto com o binômio desde a atenção básica (BARBOSA MAM, et al., 2009; CARVALHO ZMF, et al., 2017). O enfermeiro realiza, portanto, papel de mediador e facilitador do relacionamento entre paciente, família e equipe (CARVALHO ZMF, et al., 2017). 
A partir da consulta de enfermagem, o enfermeiro percebe, por meio do vínculo construído, o contexto saúde/doença da família, planeja e implementa as ações de promoção, prevenção, recuperação e reabilitação. Por meio da escuta, percebe os problemas existentes para orientar a família e contribuir significativamente para suas soluções (SOUZA TV, et al., 2020).

A partir de então, pode compartilhar com os outros membros da equipe de saúde e potencializar a assistência à pessoa e à família, desenvolvendo então uma assistência integral e compartilhada (ANDRADE LT, et al., 2010; FARO ACM, 2006). De acordo com Andrade LT, et al. (2010), nesse sentido, o papel do enfermeiro é descrito como "em expansão".

\section{Enfermeiro como gerente da equipe de Enfermagem}

Conforme Bitencourt NR, et al. (2012), as intervenções de enfermagem, dentro da própria equipe ou dentro da equipe multiprofissional devem ter sempre como foco o compromisso ético e o elo entre a pessoa deficiente e os demais membros da equipe.

O enfermeiro como supervisor da equipe de enfermagem é responsável pela comunicação, gerenciamento de conflitos, tomada de decisão, resolução de problemas e articulação com os diversos níveis de atenção dentro da rede de atenção à pessoa deficiente (ANDRADE LT, et al., 2018; FARO ACM e LEITE VBE, 2005).

Dessa forma trabalhos como o de Faro ACM e Leite VBE (2005) trazem o enfermeiro dentro da equipe como líder, já que realiza papel de supervisão e orientação, organiza escalas de folga, distribui e dimensiona o pessoal. Além disso, verifica e controla o processo de enfermagem, promove o vínculo terapêutico entre a equipe entre si e com o paciente.

$\mathrm{Na}$ equipe multiprofissional, o enfermeiro exerce ainda função de articulador do processo de trabalho em saúde, através do planejamento de reuniões de treinamento e aprimoramento, bem como a avaliação do funcionário admitido na equipe de reabilitação, o que acaba por desempenhar, por vezes, o planejamento geral da reabilitação (FARO ACM e LEITE VBE, 2005).

O Ministério da Saúde brasileiro (BRASIL, 2017) menciona que o processo de reabilitação prenuncia uma abordagem interdisciplinar com o envolvimento de profissionais, cuidadores e familiares na atenção à saúde da pessoa com deficiência.

Conforme Alves LR, et al. (2019), o enfermeiro gerente não somente administra o cuidado de enfermagem, mas integra áreas administrativas, assistenciais e de ensino/pesquisa, para qualificar a assistência e proporcionar condições e ambiente favorável para sua execução, bem como para o bem-estar do cliente. Sendo assim, desempenha função de articulador para operacionalizar o sistema de saúde, os serviços e a assistência à saúde (ALVES LR, et al., 2019).

Todavia, como apontado por Andrade LT, et al. (2010), embora o enfermeiro seja um ser fundamental dentro da equipe, dificilmente é enxergado como tal, já que sua própria falta de visão e percepção do seu papel na equipe, termina por gerar subvalorização e marginalização deste profissional pelos demais membros.

\section{CONSIDERAÇÕES FINAIS}

Foram identificados seis campos de atuação do enfermeiro no que diz respeito à área de reabilitação. $O$ desenvolvimento dessa pesquisa possibilitou uma análise da atitude dele nesse contexto, que como membro da equipe multiprofissional, emerge com papel expressivo junto aos demais membros, já que conforme trazido pela literatura, atua na educação/formação, na implementação do cuidado direto, no gerenciamento da equipe, na esfera sociopolítica, na mediação com o paciente, família e equipe multiprofissional, e na pesquisa. Esta produção contribui para o campo de trabalho em Enfermagem, quanto às reflexões acerca da inserção, valorização e reconhecimento do enfermeiro nos serviços de reabilitação. 


\section{REFERÊNCIAS}

1. ALMEIDA PC, et al. Qualidade de vida em pacientes com lesão medular. Revista Gaúcha de Enfermagem, 2013; 34(1):155-163.

2. ALVES LR, et al. Contribuições e desafios do gerenciamento de enfermagem hospitalar: evidências científicas. Revista Gaúcha de Enfermagem, 2019; 40.

3. ANDRADE LT, et al. Papel da Enfermagem na reabilitação física. Revista Brasileira de Enfermagem, 2010.63 (6): 1056-60.

4. ARAÚJO CAC, CALDAS AJ. A práxis da enfermagem de reabilitação e os contributos da osteopatia. Revista de Enfermagem Referência, 2020; 5(1): e19076.

5. ALVAREZ AB. A construção e validação de um aplicativo deEnfermagem de reabilitação voltado a pessoas com lesão medular e seuscuidadores sobre prevenção e tratamento de lesões por pressão. Tese (Doutorado em Enfermagem) - Escola de Enfermagem Anna Nery. Universidade Federal do Rio de Janeiro, Rio de Janeiro, 2018.

6. ANDRADE LT. Catálogo CIPE® para pacientes adultos em processo de neurorreabilitação. Tese (Doutorado em Enfermagem) - Escola de Enfermagem. Universidade Federal de Minas Gerais, Belo Horizonte, 2018.

7. AZEVEDO PMDS, et al. Dependência funcional na alta dos cuidados intensivos: relevância para a enfermagem de reabilitação. Revista de Enfermagem Referência, 2019; 4(20).

8. BADENOCH D, et al. Oxford Centre for Evidence-Based Medicine - Levelsofevidence, 2019.

9. BARBOSA MAM, et al. Cuidado da criança com deficiência: suportesocial acessado pelas mães. Revista Gaúcha de Enfermagem, 2009;30(3):406-12.

10. BITENCOURT RN, et al. Mudanças na vida das pessoas com lesão medular adquirida. Revista Eletrônica de Enfermagem, 2012; 14(1):95-103.

11. BOELL JEW, et al. Viver com Deficiência Física e o papel da rede de apoio. Revista de Enfermagem do Centro-Oeste Mineiro, 2018; 8: e1957.

12. BRASIL. Ministério da Saúde. Saúde da Pessoa com Deficiência: diretrizes, políticas e ações. 2017

13. CABRAL IE, NEVES ET. Cuidar de crianças com necessidades especiais de saúde: desafios para as famílias e enfermagem pediátrica. Revista Eletrônica de Enfermagem, 2009;11(3):527-38.

14. CALDATO VG, et al. Diagnósticos de enfermagem para o planejamento da alta de homens prostatectomizados: um estudo preliminar. Revista Eletrônica de Enfermagem, 2009;11(2):286-94.

15. CAMANHO GL. Nível de evidência. Revista Brasileira de Ortopedia, 2009;44(6).

16. CARVALHO ZMF, et al. Perfil epidemiológico das crianças com paralisia cerebral em atendimento ambulatorial. Revista Enfermagem UERJ, 2017; 25: e8780.

17. CARVALHO R, et al. Revisão integrativa: o que é e como fazer. Einstein (São Paulo), 2010; 8(1).

18. COUTO GR, et al. Terapêuticas de enfermagem na pessoa com deglutição comprometida após acidente vascular cerebral. Revista de Enfermagem Referência, 2019;4(23).

19. DUPAS G, et al. Identificando necessidades de crianças com deficiência auditiva: uma contribuição para profissionais da saúde e educação. Escola Anna Nery, 2011; 15(4):791-801.

20. ECHEVERRÍA LG, et al. Vivencia de discapacidad por traumatismo de la medula espinal y elproceso de rehabilitación. Ciencia y enfermeria, $2011 ; 17(1): 81-94$.

21. FARIA ACA, et al. Percurso da pessoa com acidente vascular encefálico:do evento à reabilitação. Revista Brasileira de Enfermagem, 2017; 70(3):495-503.

22. FARO ACM, LEITE VBE. O cuidar do enfermeiro especialista em reabilitação físico-motora. Revista da Escola de Enfermagem da USP, 2005; 39(1):92-6.

23. FARO ACM. Enfermagem em Reabilitação: ampliando os horizontes, legitimando o saber. Revista da Escola de Enfermagem da USP, 2006; 40(1):128-33.

24. FERGUSSON MEM, REY MCPA. Cuerpo y corporalidad em la paraplejia: significado de los câmbios. Avances en enfermaría, 2012; 30(1).

25. FRANÇA ISX, PAGLIUCA LMF. Inclusão social da pessoa com deficiência: conquistas, desafios e implicações para a enfermagem. Revista da Escola de Enfermagem da USP, 2009; 43(1):178-185.

26. IBGE- Instituto Brasileiro de Geografia e Estatística. Censo Demográfico 2010. Características gerais da população, religião e pessoas com deficiência. Rio de Janeiro, 2010;114p.

27. LEITE JL, et al. Enfermeiro pesquisador e enfermeiro assistencial: construção e projeção de identidades polimorfas. Revista Brasileira de Enfermagem, 2019; 72 (Supl1).

28. LEOPARDI MT, et al. Pesquisa em Enfermagem de Reabilitação: apontamentos da realidade brasileira. In: GOMES, B, et al. Investigação em Enfermagem de Reabilitação. Escola Superior de Enfermagem do Porto. Porto, 2014; 3645p.

29. MANCUSSI AC. Assistência ao binômio paciente/família na situação de lesão traumática da medula espinhal. Revista Latino-americana de Enfermagem, 1998; 6(4):67-73.

30. MAURICIO VC. A pessoa estomizada e o processo de inclusão no trabalho: contribuição para a enfermagem. 2011. 171 f. Dissertação (Mestrado em Enfermagem) - Faculdade de Enfermagem. Universidade do Estado do Rio de Janeiro, Rio de Janeiro, 2011; 171p.

31. UN NEWS. 2018. In: First-ever UN report on disability and development, illustrates inclusion gaps. Disponível em:<https://news.un.org/en/story/2018/12/1027311>. Acesso em: 3 nov. 2020.

32. RAMOS NA, et al. Processo de Enfermagem para pacientes com Anorexia Nervosa. Revista Brasileira de Enfermagem, 2011;64(1): 193-7.

33. SALES JNF, et al. A enfermagem no cuidado com o idoso portador de Alzheimer. Revista Eletrônica Acervo Saúde, $2019 ; 18$.

34. SOUZA TV, et al. Modelos teóricos utilizados por enfermeiros para avaliação da família: reflexão teórica. Revista Eletrônica Acervo Saúde, 2020; 12(4). 